\title{
Miocardiopatía de Tako-Tsubo presentación atípica
}

\section{Tako-Tsubo cardiomyopathy atypical presentation}

\author{
Damaris Baculima Gutiérrez ${ }^{1}$, Melissa Cárdenas Vásconez ${ }^{1}$, José Ortiz Cumbe ${ }^{1}$, Laura Miranda Ojeda ${ }^{1}$ y Lizette \\ Espinosa Martí ${ }^{1 *}$ \\ ${ }^{1}$ Universidad Católica de Cuenca \\ *lisyespinosa68@gmail.com
}

DOI: https://doi.org/10.26871/killkana_salud.v4i2.281

\begin{abstract}
Resumen
Contexto: La miocardiopatía de Tako-tsubo (STK) afecta a pacientes principalmente del género femenino, posterior a un evento estresante físico o psicológico, asociado a una clínica de dolor precordial con cambios electrocardiográficos, alteraciones transitorias de contractilidad de predominio anteroapical del ventrículo izquierdo (VI), con leve aumento de marcadores de daño miocárdico y ausencia de evidencia angiográfica de oclusión de vasos coronarios agudos como mecanismo desencadenante del cuadro. Objetivo: Presentar el caso clínico de una paciente femenina de 73 años con personalidad colérica, que ingresó a emergencias con disnea severa y cianosis generalizada. Metodología: El Ecocardiograma y la Radiografía de Tórax se observa el abombamiento apical del Ventrículo Izquierdo (VI). El STK es una entidad poco frecuente y su etiología aun es desconocida por lo que para su diagnóstico se han implementado los Criterios Diagnósticos de la Clínica Mayo y su tratamiento es empírico y multidisciplinario. Resultados: se puede encontrar el STK en una presentación clínica de Insuficiencia Cardíaca aguda, pues la paciente cursó con un edema agudo del pulmón y shock cardiogénico, también es oportuno mencionar que debido al carácter colérico de la paciente es un criterio diagnóstico y factor predisponente para presentar esta enfermedad. Conclusiones: Al no haber recomendaciones terapéuticas claras, se ha implementado el uso de beta-bloqueadores, con la idea de contrarrestar el efecto deletéreo de las catecolaminas, así como mejorar el flujo en aquellos pacientes con la obstrucción del tracto de salida del VI (OTSVI).
\end{abstract}

Palabras clave: Cardiomiopatía de TakoTsubo, Enfermedades Raras, Estrés.

\begin{abstract}
Context: Takotsubo cardiomyopathy (TCM) affects mainly female patients following a stressful physical or psychological event; associated with a precordial pain clinic; with electrocardiographic changes, transient alterations of contractility of anteroapical predominance of the left ventricle $(L V)$; with slightly increased markers of myocardial damage and no angiographic evidence of acute coronary vessel occlusion, as a triggering mechanism for the condition. Objective: To present the clinical case of a 73-year-old female patient with a choleric personality, who entered the emergency room with severe dyspnea and generalized cyanosis. Methodology: The Echocardiogram and Chest X-ray show a left Ventricle (LV) apical bulging. STK is an uncommon entity of a still unknown etiology, so the Mayo Clinic Diagnostic Criteria have been implemented for its diagnosis. Its treatment is empirical and multidisciplinary. Results: STK can be found in a clinical presentation of Acute Heart Failure, as the patient presented acute pulmonary edema and cardiogenic shock. It is appropriate to mention that due to the patient's choleric nature, this is a diagnostic criterion and predisposing factor for presenting this disease. Conclusions: In the absence of clear therapeutic recommendations, the use of beta-blockers has been implemented, to counteract the deleterious effect of catecholamines, and to improve the flow in those patients presenting the Left Ventricular outflow tract obstruction (LVOTO).
\end{abstract}

Keywords: TakoTsubo Cardiomyopathy, Rare Diseases, Stress.

\section{Introducción}

La miocardiopatía de Tako-tsubo (STK), también conocido como "Síndrome del corazón roto" o cardiopatía de estrés o apical de bolloning, fue descrita en Japón en el año 1991 por Sato y Col, siendo la misma una entidad poco frecuente cercana al $1 \%$ que simula muchas de las características de un síndrome coronario agudo, que se describen en pacientes principalmente del género feme- nino, post menopáusicas entre los 50 y 80 años, quienes posterior a un evento estresante físico o psicológico asocian clínica de dolor precordial, con cambios electrocardiográficos (elevación del segmento ST, prolongación del intervalo QT y depresión de la onda T), alteraciones transitorias de contractilidad de predominio anteroapical del ventrículo izquierdo (VI) con leve aumento de marcadores de daño miocárdico y ausencia de evidencia angiográfica de oclusión de vasos coronarios agudos como mecanismo desencadenante 
del cuadro. La característica morfológica que adopta el ventrículo izquierdo secundario a hipocinesia o discinesia de los segmentos apicales e hipercontractilidad compensatoria de las paredes basales, con fracción de eyección baja. A diferencia del síndrome coronario agudo, los pacientes con disfunción del ventrículo izquierdo no presentan enfermedad aterotrombótica en las arterias coronarias; además, las alteraciones son de carácter reversibles, tanto: clínicas, analíticas, electrocardiográficas y ecocardiográficas. ${ }^{1-3}$

El sello distintivo del STK es la discinesia anteroapical con hipercinesia basal del VI la disfunción es severa, característicamente reversible, siendo de pronóstico generalmente bueno, suele variar desde dolor, insuficiencia cardiaca, choque cardiogénico hasta, la muerte; el trastorno contráctil regresa a la normalidad entre una a tres semanas. ${ }^{4,5} \mathrm{El}$ tratamiento, empíricamente, es similar al del infarto agudo de miocardio, con especial atención en la introducción de betabloqueantes y anticoagulación. Aunque durante el momento agudo es una patología no exenta de complicaciones, predominando la insuficiencia cardíaca, edema agudo del pulmón y shock cardiogénico; a largo plazo tiene buen pronóstico y su recurrencia es rara. 6,7

\subsection{Criterios de la Clínica Mayo para el diagnóstico del Sindrome de Tako-tsubo}

1) Disfunción temporal de la contractilidad del VI, caracterizada por hiperkinesia, hypokinesia o diskinesia, de carácter regional que se extiende más allá de la distribución de una simple arteria coronaria. Raramente es focal o global.

2) Ausencia de enfermedad obstructiva coronaria o no evidencia de ruptura aguda de placa coronaria.

3) Hallazgo de nuevas alteraciones electrocardiógrafica (elevación del segmento ST con o sin cambios en la onda T) o modesta elevación de enzimas cardíacas.

4) Ausencia de feocromocitoma o miocarditis, u otras causas conocidas de disquinesia ventricular.

\section{CASO CLÍNICO:}

Se reporta el caso de paciente femenina de 73 años de edad, Diabética hace 12 años para lo cual usa metformina 850 mg vía Oral cada día, insulina Humana de Acción lenta 12 unidades al día; Hipertensa con tratamiento de enalapril, micardis plus y Ácido Acetil Salicílico diariamente, además de atenolol que no toma de forma regular; con un Hipotiroidismo subclínico tratada con levotiroxina que no refiere dosis; Insuficiencia Renal crónica y colocación de marcapaso bicameral hace 4 años. Según familiares la paciente presenta personalidad colérica, por lo que una hora y media antes de acudir a emergencias del Instituto Ecuatoriano de Seguridad Social de Cuenca, tuvo un inconveniente doméstico y de forma súbita comienza con falta de aire a pequeños esfuerzos, disnea que progresa por lo que es trasladado para su ingreso con una disnea severa y cianosis generalizada.

\section{EXAMEN FÍSICO:}

TA: 107/37 F.C.: 58 x min F.R: 44 x min SAT O2: $50 \%$ FIO2: $21 \%$ para lo que se administra oxigenoterapia dando una SAT O2: $60 \%$ CON FIO2 AL $60 \%$. Cianosis generalizada. Aparato Respiratorio: estertores crepitantes diseminados en ambos campos pulmonares. Abdomen: se evidencia equimosis a nivel periumbilical. Miembros inferiores: presencia de livideces. Región Inguino-genital no valorable. Examen neurológico: Vigil, orientada en tiempo espacio y persona.

Paciente en malas condiciones generales e inestable hemodinámicamente. Bajo normas de asepsia y antisepsia se realiza colocación de Tubo Endotraqueal sin complicaciones con obtención de secreciones sanguinolentas en abundante cantidad. Es admitida en Unidad de Cuidados Intensivos (UCI), intubada con soporte ventilatorio mecánico, se mantiene en mal estado general con SAT $0276 \%$ con $\mathrm{FIO} 2100 \%$ cianosis distal, Campos Pulmonares con estertores crepitantes abundantes bilaterales, pulso débil con FC de $60 \times$ min

\section{EXÁMENES DE LABORATORIO:}

Glicemia capilar: $7.22 \mathrm{mmol} / \mathrm{L}$. Examen de sangre: evidencia de serie blanca alterada (leucocitosis con neutrofilia), química sanguínea con enzimas cardiacas elevadas (CK-MB), glucosa central de $22.31 \mathrm{mmol} / \mathrm{L}$, creatinina ligeramente elevada $(185.64 \mu \mathrm{mol} / \mathrm{L})$ y $\mathrm{PCR}>100 \mathrm{mg} / \mathrm{l}$. Gasometría Arterial: acidosis metabólica.

\section{DATOS DE GABINETE:}

EKG: inversión de onda "T" (Imagen 1); Rx Tórax (Imagen 2): donde se evidencia infiltrado en alas de mariposa bilateral. Ecocardiograma: (Imagen 3); alteración de la contractilidad segmentar del Ventrículo Izquierdo con hipocinesis anterior, discinesia apical, fracción de eyección del Ventrículo Izquierdo 44 \% (SIMPSON). Hemodinámica del día: paciente con edema agudo del pulmón y choque cardiogénico súbito; sometida a cateterismo de urgencia que muestra coronarias normales y disfunción apical severa del Ventrículo Izquierdo. Diagnóstico de Miocardiopatía Tako-tsubo

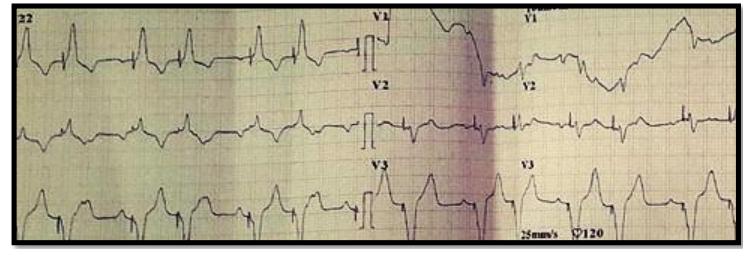

Imagen 1. 


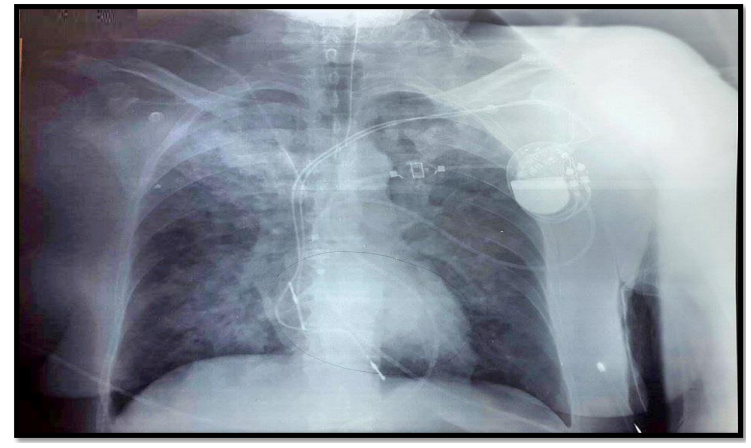

Imagen 2.

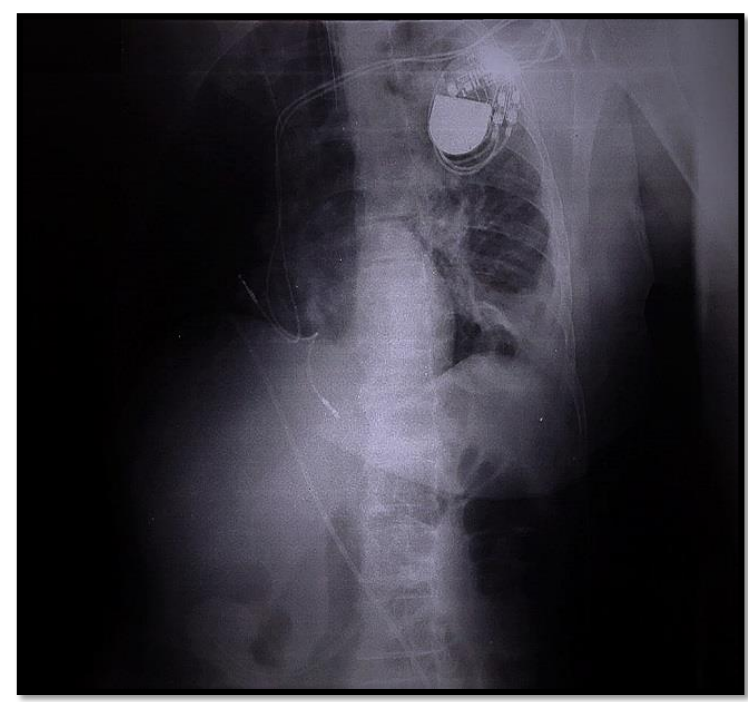

Imagen 3.
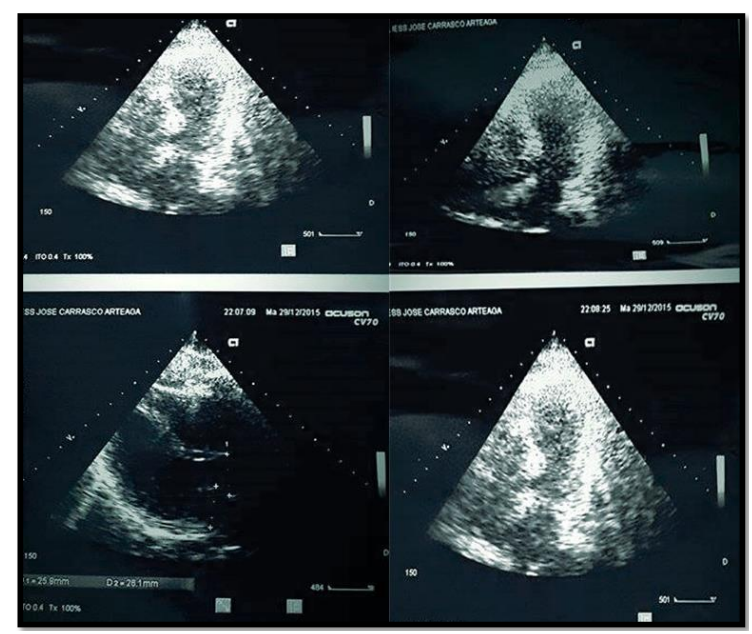

Fig. 4: Imagen 4.

\section{Evolución}

Paciente luego de 6 horas en la emergencia es trasladada a la Unidad de Cuidados intensivos (UCI), en donde sus primeros 6 días tiene pronóstico reservado, con un estado muy delicado permaneciendo con ventilación mecánica y sedación, en el que presenta en sus inicio acidosis metabólica, picos de hiperglicemia, se procedió a colocar un catéter venoso central, fue administrado un soporte vasopresor para compensar su cuadro. Durante su estancia en UCI presentó picos febriles de hasta $39.5^{\circ} \mathrm{C}$ acompañados de leucocitosis y neutrofilia. En su décimo día la paciente se encuentra hemodinámicamente estable apoyada con norepinefrina, la cual se ha ido disminuyendo las dosis progresivamente al igual que la dobutamina, en lo neurológico se disminuye sedación para mantener Ramsay de 3 y 4, la función renal permanece con hiperazoemia pero con electrolitos dentro de parámetros normales, en su último día se retira la ventilación mecánica y se hace traslado a sala, donde permanece sin novedades ni secuelas, con pronóstico favorable y posterior alta sin secuelas cardiovasculares relacionadas con el cuadro descrito.

\section{Discusión}

El STK se engloba clínicamente en ocasiones en un grupo más amplio que comprende según la American Heart Association (AHA) los conocidos como síndromes coronarios agudos sin lesiones significativas en el angiograma coronario, conocido también como Síndrome de discinesia apical transitoria (TakoTsubo) que simula un infarto agudo de miocardio; fue descrito por primera vez en 1991 por los japoneses Sato y Dote. ${ }^{4,8,9}$ Esta situación puede cursar muy raramente con complicaciones como shock cardiogénico y edema pulmonar, se presume que entre el 7 y el $32 \%$ de las mujeres postmenopaúsicas y entre el 6 y el $12 \%$ de los varones que ingresan con sospecha de infarto pueden presentar STK; comienza como un cuadro clínico caracterizado por dolor precordial tipo opresivo presente entre el 66-90\% de los pacientes, que se acompaña de disnea o simplemente puede presentarse como un episodio de sincope. ${ }^{10-13} \mathrm{Su}$ diagnóstico se ve caracterizado en el electrocardiograma por elevación del ST-T en precordiales y datos analíticos de infarto como elevación de troponinas y Creatinkinasa, pero sin datos de obstrucción coronaria en la angiografía es decir hay viabilidad de las arterias, acompañada de afectación reversible en la contractilidad en la región antero-apical del ventrículo izquierdo y un balonamiento del ventrículo izquierdo en sístole. Sin duda hasta la realización de una coronariografía con ventriculografía (prueba diagnóstica de elección) se debe de iniciar tratamiento como si se tratase de un Infarto Agudo del Miocardio (IAM) y nunca olvidar descartar la Miocardiopatía inducida por cocaína que no es el caso de la paciente por la edad y no existir antecedentes, pero debe siempre considerarse como un diagnóstico diferencial significativo en pacientes con esta patología. ${ }^{1,12,14,15}$

En nuestro caso expuesto, podemos encontrar el STK en una presentación clínica de Insuficiencia Cardíaca aguda ya que aparte de presentar hipocinesia anterior y disquinesia apical del ventrículo izquierdo, inversión de la onda "T" 
en el electrocardiograma, elevación de troponinas y creatininkinasa en la química sanguínea, la paciente cursó con un edema agudo del pulmón y shock cardiogénico, también es oportuno mencionar que debido al carácter colérico de la paciente es un criterio diagnóstico y factor predisponente para presentar esta enfermedad; el diagnóstico fue confirmatorio cuando al realizarse la colocación del catéter y exploración coronarias, mostró viabilidad de las arterias coronarias, además que al realizar los siguientes exámenes de gabinete no hubo presencia de necrosis cardiaca en el electrocardiograma y su pronóstico fue favorable por ser una entidad reversible y sin mayores complicaciones que las que presenta en su modo atípico. ${ }^{1,2,14,16}$ Al revisar la bibliografía encontramos que en Perú hubo un caso descrito de Tako-Tsubo con las mismas características clínicas, electrocardiográficas y ecocardiografías muy similares a las expuestas en el caso detallado, de igual manera la paciente cursó con shock cardiogénico y lo particular de los dos, es que en ambos casos, sus complicaciones fueron reversibles y evolucionaron favorablemente, también se recalca que ambas pacientes estuvieron ingresadas en UCI como se indican en las literaturas, al ser importante el manejo completo y eficaz de las complicaciones que suele cursar con STK.. ${ }^{1,2,17}$

Al no haber recomendaciones terapéuticas claras, se ha implementado el uso de betabloqueadores, con la idea de contrarrestar el efecto deletéreo de las catecolaminas, así como mejorar el flujo en aquellos pacientes con la obstrucción del tracto de salida del VI (OTSVI). Por similitud en el tratamiento con el feocromocitoma, en el que se asocian alfa-bloqueantes y beta-bloqueantes, se podría considerar adecuado el tratamiento con carvedilol. En estos pacientes los inhibidores de la enzima convertidora de angiotensina no han demostrado beneficios en el remodelado ventricular. La anticoagulación está indicada en pacientes con trombo intracavitario, con la intención de prevenir los fenómenos embólicos. En algunos casos, durante la presentación clínica, la situación hemodinámica puede ser tan delicada que el paciente requiera aporte de líquidos, fármacos vasoactivos e inotrópicos como fenilefrina, y levosimendán, intubación orotraqueal e incluso balón de contrapulsación u otro tipo de soporte mecánico. El pronóstico es favorable, sin embargo la mortalidad intrahospitalaria va del $2 \%$ al $4 \%$, debido a complicaciones fatales como arritmias malignas, ruptura de pared libre del VI y choque cardiogénico del $9 \%$ al $20 \%$, por OTSVI ó por fallo de bomba. ${ }^{16,18}$

\section{Fuente de Financiamiento}

Este estudio es autofinanciado.

\section{Conflicto de Intereses}

No existen conflictos personales, profesionales, financieroso de otro tipo.

\section{Consentimiento Informado}

Los autores cuentan con el consentimiento informado de los pacientes para la investigación, la publicación del caso y sus imágenes.

\section{Referencias Bibliográficas}

1. Warner L, Loscalzo J. Miocardiopatía y miocarditis. In: Longo D, Fauci A, Kasper D, et al, editors. HARRISON principios de Medicina Interna. 18th ed. México: Mc Gram Hill; 2012. p. 1964.

2. Bosch X. Cardiopatía Isquémica. In: Rozman C, Cardellach F, Farreras-Rozman, editors. Medicina Interna. XVII ed. Barcelona: ELSEVIER; 2012. p. 443.

3. Ramírez Chaves JJ, Calderón Calvo C. Miocardiopatía tipo Takotsubo. Revista Costarricense de Cardiología. 2014 06;16:25 - 32.

4. Rau CM, Kauffmann M, Rau CL, Cereceda M, Castro G, Massardo T. Síndrome de Tako-Tsubo: caso clínico. Revista médica de Chile. 2011 03;139:348 - 352.

5. García E, Restrepo G, Cubides CA, Múnera AG, Aristizábal D. Miocardiopatía por estrés (miocardiopatía tipo takotsubo): presentación de un caso clínico y revisión de la literatura. Revista Colombiana de Cardiología. 2006 08; $13: 31-38$.

6. Núñez-Gil IJ, Molina M, Bernardo E, Ibañez B, RuizMateos B, García-Rubira JC, et al. Síndrome de takotsubo e insuficiencia cardiaca: seguimiento a largo plazo. Revista Española de Cardiología. 2012 Nov;65(11):9961002.

7. Aguirre DM, Mejía DT, Atehortua DFL, Florez JS, Vasquez EVO. Enfermedad del corazón roto o síndrome de Tako Tsubo. Médicas UIS. 2011;24(1).

8. Núñez-Gil IJ, Luaces-Méndez M, García-Rubira JC. Cardiopatía de estrés o síndrome de Tako-Tsubo: conceptos actuales. Revista argentina de cardiología. 2009;77(3):218-223. Recuperado en 28 de julio de 2020, de.

9. FORTUNA L, HAMITY L, ALDAY L, CONTRERAS A, TIBALDI M, MOREYRA E, et al. SÍNDROME DE TAKOTSUBO EXPERIENCIA EN TRES INSTITUCIONES DE LA CIUDAD DE CÓRDOBA. Medicina (Buenos Aires). 2014;74(1).

10. Segura-Pujal L. El síndrome de Tako-tsubo: un diagnóstico fallido. Revista Archivo Médico de Camagüey. 2010;14(5).

11. Araújo Maciel B, de Lima Cidrão AA, dos Santos Sousa tB, da Silva Ferreira JA, Messias Neto VP. Pseudoinfarto agudo do miocárdio devido à síndrome da disfunção ventricular apical transitória (síndrome de Takotsubo). Revista Brasileira de Terapia Intensiva. 2013 03;25:63 67.

12. Jiménez SR, Valle JSL. Cardiomiopatía de Takotsubo, el gran imitador del infarto agudo del miocardio. Revista CES Medicina. 2012;26(1):107-120. 
13. Sánchez Llanos P, Sánchez Hernández C, García Alonso R, Río Cordová Id. Síndrome de Tako Tsubo. Revista Clínica de Medicina de Familia. 2010 06;3:127 - 130.

14. Molero Silvero E, Borrego Jiménez P, Bartolomé Cela E, Rada Martínez I, Carretero Quevedo A. Síndrome de Takotsubo: a propósito de un caso. Sanidad Militar. 2012 09;68:168 - 171 .

15. del Nogal Sáez F, García-Sípido EM, Molina MV, Navalpotro $\mathrm{MnB}$, Abad RD, Ingelmo JG. Síndrome de tako-tsubo: discinesia transitoria del ventrículo izquierdo. Presentación de nuestra casuística. Medicina intensiva. 2011;35(5):307-311.

16. del Solar-Moreno Z, Ulloa-Montoya H, Orellana-Cuellar L. Recurrencia de síndrome de Tako-Tsubo: un caso peruano. Revista médica de Chile. 2015 08;143:1085 1086.

17. Ramírez Chaves JJ, Calderón Calvo C. Miocardiopatía tipo Takotsubo. Revista Costarricense de Cardiología. 2014 06;16:25 - 32.

18. Levin R, Degrange M, Jiménez A, Porcile R. Balón de contrapulsación e inotrópicos no catecolamínicos en el manejo del shock cardiogénico secundario a cardiopatía de Takotsubo. Una serie de casos. Insuficiencia cardíaca. 2015;10(4):164-168.

Recibido: 12 de febrero de 2020

Aceptado: 15 de junio de 2020

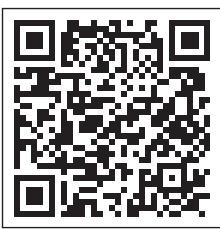


